\title{
Feasibility study of Zeeman modulation spectrometry with a hollow capillary fiber based gas cell
}

\author{
Andreas Hangauer, ${ }^{1,2, *}$ Jia Chen, ${ }^{1,2,3}$ Rainer Strzoda, ${ }^{2}$ and Markus-Christian Amann ${ }^{1}$ \\ ${ }^{1}$ Walter Schottky Institute, Technical University of Munich, 85748 Garching, Germany \\ ${ }^{2}$ Siemens Corporate Research \& Technologies, Otto Hahn-Ring 6, 80200 Munich, Germany \\ ${ }^{3}$ Now at School of Engineering and Applied Science, Harvard University, Cambridge, Massachusetts 02138, USA \\ ${ }^{*}$ Corresponding author: andreas.hangauer@wsi.tum.de
}

Received November 7, 2011; revised February 20, 2012; accepted February 20, 2012; posted February 22, 2012 (Doc. ID 157728); published March 30, 2012

\begin{abstract}
For paramagnetic gases (e.g., $\mathrm{O}_{2}, \mathrm{NO}, \mathrm{NO}_{2}, \mathrm{OH}$ ) Zeeman modulation spectrometry is a method for spectrometric gas sensing with extraordinary selectivity. In this Letter it is combined with a hollow capillary based gas cell, where the gas is filled in long light-guiding capillary that is placed inside a toroidal coil. Over conventional Zeeman spectrometry this has the advantage of lower power consumption at long optical path length, since several loops of the hollow capillary fiber can be placed in the coil. Compared to wavelength modulation spectrometry the advantage is insensitivity to interference by multimode propagation in the fiber and absorption by other nonparamagnetic gases, which should enhance both sensor stability and sensitivity. Experimental and theoretical results are presented, showing the feasibility of the approach. (c) 2012 Optical Society of America
\end{abstract}

OCIS codes: $280.4788,300.6260$.

Zeeman modulation spectrometry (ZMS) or zero-field laser magnetic resonance (LMR) is a spectrometric detection method for paramagnetic gases, i.e. gases that show a Zeeman splitting of absorption lines if a magnetic field is applied (e.g., $\mathrm{NO}, \mathrm{NO}_{2}, \mathrm{O}_{2}, \mathrm{OH}$ ). In this technique the gas absorption line center wavelength is modulated by the magnetic field instead of the emission wavelength of a tunable laser [1,2]. Even though this method is old-invented when room temperature tunable lasers were not available in the midinfrared-it has two advantages. First, it is insensitive against other nonparamagnetic gases (e.g., $\mathrm{H}_{2} \mathrm{O}$ vapor) [3], i.e. presence of these gases does not cause a ZMS signal and second, it is also insensitive to fringes generated by multipath propagation inside the gas cell that typically limit the detection sensitivity in TDLAS (tunable diode laser absorption spectrometry) sensors. Hence, a superior sensor stability -in the sense of low baseline drift-is expected for Zeeman modulation spectrometry, as observed in the technically similar Faraday rotation spectrometry (FRS) [4,, 5$]$. In the presence of a magnetic field in direction of light propagation the absorption line will split into two or more separate lines with a spectral separation proportional to the magnetic field strength (linear Zeeman effect). A comparison between the setups for ZMS and wavelength modulation spectrometry (WMS) is shown in Fig. 1 . The even harmonic spectra for ZMS and WMS (e.g., second-harmonic) are the same if the Zeeman splitting is in two separate lines whereas ZMS does not generate odd harmonic spectra (e.g., first harmonic). This can be understood if the individual split Zeeman lines are considered separately. Each component alone would generate the same harmonic spectrum as WMS because it makes no difference whether the absorption line is moving sinusoidally or the laser emission wavelength. The WMS modulation amplitude corresponds to the amplitude of the Zeeman shift. For even order harmonic spectra the sign of the modulation amplitude (for ZMS this is whether the line is shifting to higher or lower wavelength for a given magnetic field) has no influence on the spectral shape and odd harmonics appear inverted around the $y$ axis. Hence, in total, for odd orders the individual components cancel out and for even orders a superposition of WMS harmonic spectra of (possibly) different modulation index is observed.

The modulation amplitude that gives maximum signal for a single Doppler broadened line is 2.11 times the line half-width [6]. The Doppler linewidth for lines in the $\mathrm{O}_{2}$ A-band is around $420 \mathrm{MHz}$ for room temperature. Assuming an average splitting coefficient of $7 \mathrm{MHz} / \mathrm{mT}$, a magnetic field strength amplitude of $126 \mathrm{mT}$ would be needed. A lower modulation amplitude results in an (approximately) linear decrease in signal amplitude. The situation improves in the midinfrared where, for NO detection, only $18 \mathrm{mT}$ would be needed due to the reduced Doppler linewidth of $62 \mathrm{MHz}$.

With an elementary calculation (see below) it can be shown that the power consumption at a given magnetic field strength is inherently linked to the physical dimensions of the coil. It is important to have a small inner coil diameter (where the gas-cell is located) and short coil length, which is in conflict with long optical pathlength.

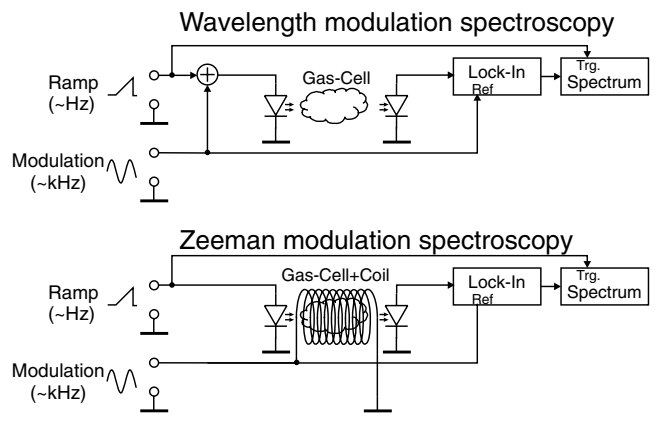

Fig. 1. Schematic setup of wavelength and Zeeman modulation systems. The slow current ramp is used to scan the spectrum by laser tuning. The faster modulation of wavelength or the magnetic field and corresponding demodulation with the lock-in amplifier realizes a derivative-like spectrum. 
To enable long optical pathlength under low power consumption, hollow capillary fiber hollow core fiber (HCF) is applied as gas cell. Recently, these HCFs were investigated for their suitability as an alternative to multipass cells [7]. They provide long optical path length at simultaneously extremely low volume (mL range). The drawback is the spectral background, given by optical interference due to highly multimode character of the fiber. Although the spectral background could be greatly reduced by applying mechanical vibration [7], it still limits the sensitivity in wavelength modulation systems. For Zeeman modulation, the spectral background of the cell is inherently moved, because the AC signals (second harmonic intensity variation in this case) are generated only by the Zeeman modulation of the gas absorption line. Unfortunately, FRS cannot be applied here, since the HCF is not polarization maintaining and a reliable detection of a rotation in polarization angle is impossible.

Since the HCF can be wound up and consumes only very little volume it can easily be placed in a toroidal air coil with small inner hole diameter. This keeps both the coil length (toroid circumference) and also its inner diameter small, which is required for low electrical power consumption (see calculation below). The experimental setup is shown in Fig. 2 . The used HCF is shown in Fig. 3

To the knowledge of the authors no study has been done on the necessary power consumption and its fundamental limits, neither for LMR, ZMS, nor FRS. This is however of great importance for possible sensor applications. When designing the coil the relevant parameters are number of windings $n$, wire diameter $d_{w}$ and dimensions of the coil like diameter of the toroid $D$, diameter of the windings $d_{s}$, and diameter of the hole where the fiber is inside $d_{i}$ (see Fig. 3).

The total volume of the copper wire forming the coil can be expressed in two ways: first by the volume of the toroid and second the length of the wire times the cross section of the wire, which gives

$$
F \frac{\pi^{2}}{4}\left(d_{s}^{2}-d_{i}^{2}\right) D=\frac{\pi^{2}}{8} n\left(d_{s}+d_{i}\right) d_{w}^{2} .
$$

Here $F$ is the filling factor, which depends on the type of winding. Its maximum value is obtained for the orthocyclic or hexagonal style winding and is in this case $F=\frac{\pi}{2 \sqrt{3}} \approx 0.91$ [ $[$ ]. The ohmic resistance of the coil $R$ and the magnetic field strength amplitude $B$ inside the coil for electric current amplitude $I$ are given by

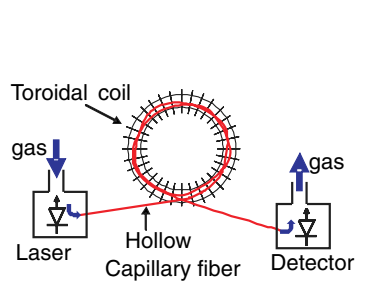

(a) Gas cell schematic

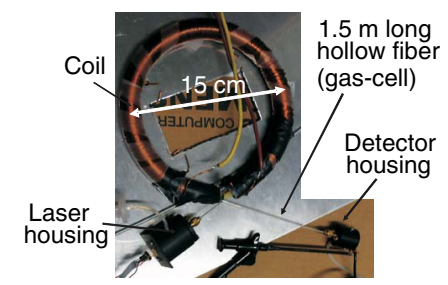

(b) Photograph
Fig. 2. (Color online) Schematic (a) and photograph (b) of the gas cell for ZMS with a HCF based gas cell.

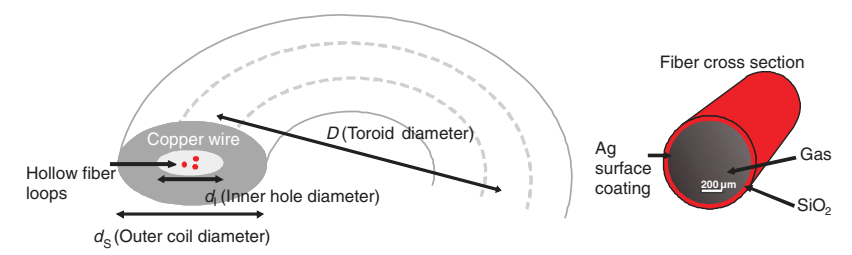

Fig. 3. (Color online) The design parameters specifying the coil dimensions. The individual winding layers of the coil are located in the dark gray area labeled "copper wire." The HCF cross section is shown on the right.

$$
R=\rho_{\mathrm{Cu}} \frac{2 n\left(d_{s}+d_{i}\right)}{d_{w}^{2}} \quad B=\mu_{0} \frac{n I}{\pi D},
$$

with $\rho_{\mathrm{Cu}}=1.68 \cdot 10^{-8} \Omega \mathrm{m}$ the resistivity of copper and $\mu_{0}=4 \pi \cdot 10^{-7} \mathrm{Tm} / \mathrm{A}$ the vacuum permeability. The dissipated power $P=R I^{2} / 2$ can be expressed in terms of $B$ by using Eq. (2) and eliminating $d_{w}$ using Eq. (1):

$$
P=\rho_{\mathrm{Cu}} \frac{\pi^{2} B^{2} D}{2 \mu_{0}^{2} F} \frac{d_{s}+d_{i}}{d_{s}-d_{i}} \approx 525 \frac{W}{T^{2} \mathrm{~cm}} \frac{B^{2} D}{F} \frac{d_{s}+d_{i}}{d_{s}-d_{i}} .
$$

If the hole diameter $d_{i}$ is large compared to the winding diameter $d_{s}$ the required power $P$ can increase dramatically, as well as if the filling factor is worse than optimum. In fact the power consumption goes to infinity if $d_{i} / d_{s} \rightarrow 1$, i.e. the coil winding layers are very thin compared to the inner hole diameter (see Table 1). This is a fundamental problem for setups where a multi- or singlepass cell is placed inside the coil (corresponds more to the $9 / 10$ case in Table 1 ). On the other hand making the winding thicker or the hole smaller $\left(d_{i} / d_{s} \rightarrow 0\right)$ the electrical power requirement still has a lower limit. Values of $d_{i} / d_{s}<1 / 3$ are already within a factor of two close to the optimum. The toroid diameter $D$ is limited by the minimum bending radius of the $\mathrm{HCF}$ and is in the range of $12 \mathrm{~cm}$ to $15 \mathrm{~cm}$. Only the use of superconductors as wire material can improve the minimum required power, in which case it goes to zero for all cases.

Another important question is whether the conductive Ag coating inside the fiber has a negative effect. First, the modulated magnetic field induced current will cause heating and second may weaken the magnetic field inside the fiber. By computing the induced voltage and the resistance of the coating, the dissipated power in the silver coating $P_{\mathrm{Ag}}$ for a fiber with length $l$, inner fiber hole diameter $d$, and coating thickness $h_{\mathrm{Ag}}$ can be expressed as

Table 1. Necessary Power for Relevant Coil Geometries (* for Present Setup) and Sinusoidal Magnetic Fields Optimum for Detection of NO (@5.3 $\mu \mathrm{m})$ and $\mathrm{O}_{2}(@ 763 \mathrm{~nm})$

\begin{tabular}{lccc}
\hline Peak Field $B$ & Toroid Diameter $D$ & $d_{i} / d_{s}$ & Power $P$ \\
\hline $18 \mathrm{mT}(\mathrm{NO})$ & $15 \mathrm{~cm}^{*}$ & 0 & $2.8 \mathrm{~W}$ \\
& & $1 / 3^{*}$ & $5.6 \mathrm{~W}$ \\
& & $9 / 10$ & $53.3 \mathrm{~W}$ \\
$126 \mathrm{mT}\left(\mathrm{O}_{2}\right)$ & & 0 & $137 \mathrm{~W}$ \\
& & $1 / 3^{*}$ & $274 \mathrm{~W}$ \\
& & $9 / 10$ & $2.69 \mathrm{~kW}$ \\
\hline
\end{tabular}




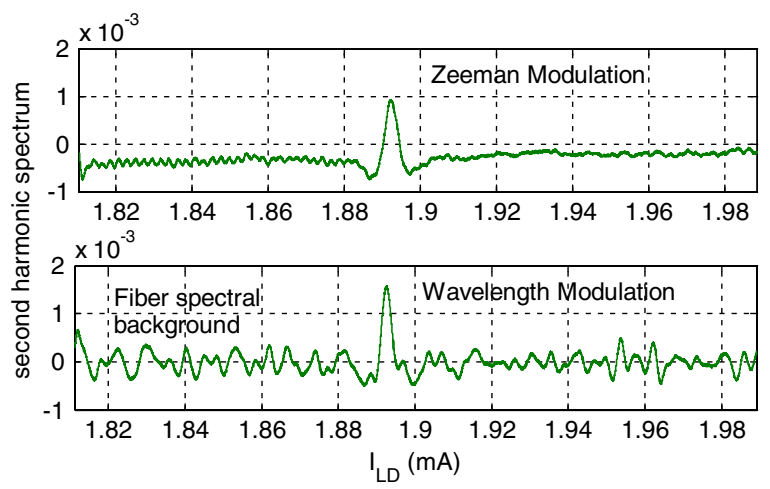

Fig. 4. (Color online) Second-harmonic spectra obtained by Zeeman modulation (top) and traditional wavelength modulation (bottom) at a $\mathrm{O}_{2}$ transition around $763 \mathrm{~nm}$. The wavelength modulation spectrum shows the fiber spectral background in the order $5 \cdot 10^{-4}$ which is not present in the Zeeman spectrum.

$P_{\mathrm{Ag}}=\pi^{3} /\left(8 \rho_{\mathrm{Ag}}\right) B^{2} f^{2} d^{3} h_{\mathrm{Ag}} l$, with $\rho_{\mathrm{Ag}}=1.59 \cdot 10^{-8} \Omega \mathrm{m}$ the resistivity of silver. For the $d=750 \mu \mathrm{m}$ fiber, assuming a typical value $h_{\mathrm{Ag}}=200 \mathrm{~nm}$ [9],$B=100 \mathrm{mT}$, and a modulation frequency $f=1 \mathrm{kHz}$ a value of $P_{\mathrm{Ag}} / l=$ $206 \mu \mathrm{W} / \mathrm{m}$ is obtained. Hence heating can be neglected. Equivalently, the generated magnetic field by the loop current in the coating is $B_{\mathrm{Ag}}=\mu_{0} \pi /\left(2 \rho_{\mathrm{Ag}}\right) f B d h_{\mathrm{Ag}}$ giving $B_{\mathrm{Ag}} / B=1.68 \cdot 10^{-5}$, i.e. the magnetic field inside the HCF is not weakened.

With the setup shown in Fig. 2, the recorded spectra in air $\left(\mathrm{O}_{2}\right.$ mixing ratio $\left.21 \%\right)$ at reduced pressure of $300 \mathrm{mbar}$ is shown in Fig. 4. The second-harmonic spectrum is recorded, which is the second-harmonic intensity divided by the DC intensity and has the unit of 1 and the values are directly proportional to absorbance. The laser (a $763 \mathrm{~nm}$ vertical-cavity surface-emitting laser) probes the $\left(J^{\prime \prime}=8, N^{\prime \prime}=9\right) \rightarrow\left(J^{\prime}=8, N^{\prime}=8\right)$ transition of $\mathrm{O}_{2}$ at $\nu=13093.66 \mathrm{~cm}^{-1}(763.73 \mathrm{~nm})$ which splits into 32 Zeeman components $[1,10]$. The laser power is around $300 \mu \mathrm{W}$ and the $\mathrm{HCF}$ length is $1.5 \mathrm{~m}$ with two loops (or $1.05 \mathrm{~m}$ of the $\mathrm{HCF}$ ) placed inside the coil. The received photocurrent is $40 \mathrm{nA}$ corresponding to a total loss of $41 \mathrm{~dB}$. This is due to the missing coating in the HCF which is a technical not a fundamental limitation because (commercially available) HCFs with proper coating have very low loss (few $\mathrm{dB} / \mathrm{m}$ ) in the visible wavelength region [11]. The modulation frequency of $f=333 \mathrm{~Hz}$ was chosen to be nonharmonically related to the $50 \mathrm{~Hz}$ line frequency. The coil with inductance $2.9 \mathrm{mH}$ and resistance $3.2 \Omega$ was operated with a current modulation amplitude of $6.1 \mathrm{~A}$ which generates a magnetic field amplitude of $B=50 \mathrm{mT}$.

In Fig. 4 the fiber spectral background with a peak to peak amplitude of $5 \cdot 10^{-4}$ is easily observed for traditional wavelength modulation with a comparable modulation amplitude. As expected, the background vanishes in the Zeeman modulated spectrum, because there only the modulation of the absorption line contributes to the optical spectrum. However, due to the high coil current and the high photocurrent amplification factor $\left(2 \cdot 10^{7} \mathrm{~V} / \mathrm{A}\right)$ a certain (time variable) electromagnetic interference (EMI) on the second-harmonic was observed. This is considered as the cause of the baseline and the small high frequency noise on the Zeeman spectrum (Fig. 4). This background depends on the magnetic field amplitude and strongly rises if the current is further increased, which is typical for such a nonlinear distortion. Nevertheless, the spectral background was reduced by a factor of four despite the existence of EMI, which can be lowered using a high transmittance fiber or using less magnetic field.

The modulation index (modulation amplitude divided by line half-width) for both WMS and ZMS is estimated to be around 0.7 by comparing the peak signal amplitude with a simulated result. The consumed power is around $60 \mathrm{~W}$ whereas the lower limit Eq. (3) predicts $43 \mathrm{~W}$. The difference is attributed to a nonoptimum filling factor and the measurement inaccuracy of the magnetic field strength.

In conclusion, we demonstrated that ZMS with a HCF is feasible. Theoretical investigations showed that power consumption is much less than for ZMS with multi- or single-pass cells because of the much smaller diameter of the coil. Future work has to quantify the sensitivity limit for the gas detection and reduction of the EMI. Theoretically the sensitivity is only limited by the laser and detector noise but not the spectral background of the cell. For quantification a fiber with dielectric coating will enhance the fiber transmission to reduce the electrical distortion on the photocurrent. Furthermore, detection of $\mathrm{NO}$ in the midinfrared is more appealing than oxygen detection in the visible, because the electrical power consumption is much less due to the reduced Doppler linewidth and hence a much lower magnetic field is needed.

\section{References}

1. W. Demtröder, Laser Spectroscopy-Basic Concepts and Instrumentation, 3rd ed. (Springer, 2003).

2. W. Urban and W. Herrmann, Appl. Phys. A 17, 325 (1978).

3. A. Kaldor, W. B. Olson, and A. G. Maki, Science 176, 508 (1972).

4. G. Litfin, C. R. Pollock, J. R. F. Curl, and F. K. Tittel, J. Chem. Phys. 72, 6602 (1980).

5. R. Lewicki, J. H. Doty, R. F. Curl, F. K. Tittel, and G. Wysocki, Proc. Natl. Acad. Sci. USA 106, 12587 (2009).

6. P. Kluczynski, A. M. Lindberg, and O. Axner, J. Quantum Spectrosc. Radiat. Transf. 83, 345 (2004).

7. J. Chen, A. Hangauer, R. Strzoda, and M. C. Amann, Appl. Opt. 49, 5254 (2010).

8. W. T. McLyman, Transformer and Inductor Design Handbook (Dekker, 2004).

9. R. George and J. Harrington, Appl. Opt. 44, 6449 (2005).

10. R. J. Brecha, L. M. Pedrotti, and D. Krause, J. Opt. Soc. Am. B 14, 1921 (1997).

11. Doko Engineering, capillary waveguide specifications, http://do-ko.jp/specs.html. 